\title{
Transcriptional oscillation of Lunatic fringe is essential for somitogenesis
}

\author{
Katrin Serth, Karin Schuster-Gossler, Ralf Cordes, and Achim Gossler ${ }^{1}$ \\ Institut für Molekularbiologie, Medizinische Hochschule Hannover, D-30625 Hannover, Germany
}

\begin{abstract}
A molecular oscillator that controls the expression of cyclic genes such as lunatic fringe $(L f n g)$ in the presomitic mesoderm has been shown to be coupled with somite formation in vertebrate embryos. To address the functional significance of oscillating $L f n g$ expression, we have generated transgenic mice expressing $L f n g$ constitutively in the presomitic mesoderm in addition to the intrinsic cyclic Lfng activity. These transgenic lines displayed defects of somite patterning and vertebral organization that were very similar to those of $L$ fng null mutants. Furthermore, constitutive expression of exogenous Lfng did not compensate for the complete loss of cyclic endogenous L fng activity. Noncyclic exogenous $L$ fng expression did not abolish cyclic expression of endogenous Lfng in the posterior presomitic mesoderm (psm) but affected its expression pattern in the anterior psm. Similarly, dynamic expression of $\mathrm{Hes} 7$ was not abolished but abnormal expression patterns were obtained. Our data are consistent with a model in which alternations of $L$ fng activity between ON and OFF states in the presomitic mesoderm prior to somite segmentation are critical for proper somite patterning, and suggest that Notch signaling might not be the only determinant of cyclic gene expression in the presomitic mesoderm of mouse embryos.
\end{abstract}

[Keywords: Somitogenesis; segmentation clock; Lunatic fringe $(L f n g)]$

Received September 30, 2002; revised version accepted February 7, 2003.

Somitogenesis is a fundamental patterning process in vertebrate embryos that subdivides the paraxial mesoderm into a metameric series of homologous subunits, the somites. Somites form sequentially by segmentation of tissues at the anterior end of the presomitic mesoderm (psm). A molecular oscillator referred to as "segmentation clock" that has been shown to be coupled with the progression of somite segmentation has been revealed by dynamic and cyclic expression of genes in the psm. Expression of cyclic genes occurs in a tightly coordinated periodicity such that one wave of expression passes through the psm during the formation of one somite (Palmeirim et al. 1997; Forsberg et al. 1998; McGrew et al. 1998; Aulehla and Johnson 1999; Jiang et al. 2000; Jouve et al. 2000). The segmentation clock is likely to be linked to Notch signaling activity as genes displaying cyclic activity encode components of the Notch pathway. Misexpression of Notch components or disruption of signaling by dominant negative factors disrupts somite formation and patterning in Xenopus and zebrafish embryos (Jen et al. 1997, 1999; Takke and Campos-Ortega 1999; Sawada et al. 2000). Furthermore, mutations in some Notch pathway components that lead to defects in somitogenesis also affect the expression of cyclic

${ }^{1}$ Corresponding author.

E-MAIL gossler.achim@mh-hannover.de; FAX 49-511-532-4283. Article and publication are at http://www.genesdev.org/cgi/doi/10.1101/ $\operatorname{gad} .250603$. genes (del Barco Barrantes et al. 1999; Jiang et al. 2000; Jouve et al. 2000; Dunwoodie et al. 2002). It has been proposed that the segmentation clock regulates the periodic activation of Notch (Pourquie 1999), its signaling is required for the synchronization of the clock in neighboring cells (Jiang et al. 2000), and the Notch pathway is part of the oscillator mechanism per se (Holley et al. 2002; Morales et al. 2002; Dale et al. 2003).

In mice, four genes, lunatic fringe (Lfng) and three bHLH genes (Hes1, Hes7, and Hey2), are known to date, which display oscillating expression in the psm (Forsberg et al. 1998; McGrew et al. 1998; Aulehla and Johnson 1999; Jouve et al. 2000; Leimeister et al. 2000; Bessho et al. 2001b). Lfng encodes a glycosyltransferase that modifies Notch in the trans-Golgi network and thereby modulates its receptiveness to various ligands (Hicks et al. 2000), and Notch signaling regulates the expression of the bHLH genes. Loss-of-function analyses have shown that Lfng and Hes 7 function are essential for normal somite formation and patterning (Evrard et al. 1998; Zhang and Gridley 1998; Bessho et al. 2001b), whereas the loss of Hes1 and Hey2 does not affect somitogenesis (Ishibashi et al. 1995; Ohtsuka et al. 1999; Jouve et al. 2000; Bessho et al. 2001b; Gessler et al. 2002). Mice homozygous for null alleles of Lfng and Hes7, respectively, display severe defects in somite compartmentalization, somites are irregular in form and size, and the vertebral column is severely disorganized (Evrard et al. 1998; Zhang and Gridley 1998; Bessho et al. 2001b). The loss- 
of-function studies of $L f n g$ and Hes7 have firmly established essential roles of these genes for somite patterning, and the apparent noncyclic expression of $L f n g$ in Hes7 mutants suggested that cyclic Lfng expression might be essential (Bessho et al. 2001a). However, thus far the significance of the oscillatory transcription of Lfng for its function during somitogenesis in mouse embryos has not been demonstrated.

To address the functional significance of oscillating Lfng activity in somite patterning, we have generated transgenic mice that express Lfng in a nonoscillating manner in the presomitic mesoderm using a portion of the Deltal promoter (msd) that directs heterologous gene expression into the paraxial mesoderm (Beckers et al. 2000|. Transgenic msd::Lfng mice had somite and vertebral column defects very similar to Lfng null mutants. Reducing the level of endogenous Lfng did not significantly alter the phenotype caused by the transgene, and the nonoscillating exogenous $L$ fng did not rescue the loss of endogenous cyclic Lfng. Nonoscillating exogenous Lfng did not block dynamic expression of endogenous Lfng in the posterior psm but lead to a diffuse broad expression domain in the anterior psm. Likewise, dynamic expression of Hes7 was not completely abolished but abnormal expression patterns were obtained. Our data provide direct experimental evidence that oscillations of Lfng transcription between active and inactive states are critical for $L f n g$ function in the paraxial mesoderm and suggest that Notch signaling might not be the sole determinant of cyclic transcriptional activation of oscillating genes in the posterior presomitic mesoderm of mouse embryos.

\section{Results}

Generation and skeletal defects of msd::Lfng transgenic mice

To analyze the effect of nonoscillating ("constitutive") Lfng transcription on somite formation and patterning, we generated transgenic mice by DNA microinjection with Lfng cDNAs encoding an untagged and a C-terminally HA-tagged $L f n g$ protein, respectively (Fig. 1A). In both cases, the $1.5-\mathrm{kb}$ msd fragment from the Delta1 gene, which directs heterologous gene expression in the presomitic mesoderm and at later stages additionally in newly formed somites and myotomes, was fused to the Delta1 minimal promoter and the 5' UTR of exon 1 up to the ATG codon (Beckers et al. 2000). The Lfng cDNAs were fused in frame to the Deltal ATG. 3' to the Lfng coding sequence an IRES sequence followed by a destabilized GFP cDNA, and polyadenylation signal was included (see Material and Methods for details).

Five transgenic founder mice with shortened and kinked tails were obtained (Fig. 1B, panels a,b; data not shown). Three carried the C-terminally HA-tagged and two carried the untagged Lfng transgene. Two male founders carrying the tagged version of Lfng and one female founder carrying the untagged Lfng cDNA bred and transmitted the transgene to the offspring. Two trans- genic lines referred to as msd::LfngHA2 and msd::LfngHA3 were established from the founders carrying the tagged $L f n g$. The female founder carrying the untagged transgene (Fig. 1B, panel b) gave rise to only one litter of stillborns with severe skeletal malformations (Fig. 1C, panel a) precluding the establishment of a stable transgenic line and the analysis of transgenic embryos. However, two phenotypically inconspicuous founders, referred to as msd::Lfng11 and msd::Lfng12, were obtained from an additional series of microinjections with the untagged Lfng transgene and gave rise to transgenic offspring with shortened and kinked tails (Fig. 1B, panel c; data not shown). The female founder msd:: Lfng12 produced four litters with 31 offspring, only two of which were transgenic and did not breed thus far. In contrast, the male founder msd:: Lfng11 transmitted the transgene consistently allowing us to collect and analyze transgenic embryos for somite defects.

Transgenic founders and hemizygous mice carrying both the HA-tagged and untagged Lfng transgene displayed similar external phenotypes and axial skeleton defects (e.g., Fig. 1B, cf. panels b and f, C, cf. panels a and e). The segmental pattern of the vertebral column was disrupted; irregularly shaped vertebral bodies and fusions of adjacent neural arches and proximal ribs were consistently obtained (Fig. 1C; data not shown). However, the severity of the phenotype varied between different founders and transgenic lines carrying either transgene. Hemizygous msd::LfngHA3 mice $(\mathrm{n}=25)$ displayed vertebral malformations and severe shortening of the body axis (Figs. 1B, panel f, C, panel e, 4A, panel c, below) similar to Lfng null mutants (Evrard et al. 1998; Zhang and Gridley 1998). In contrast, hemizygous msd::LfngHA2 mice showed milder defects $(\mathrm{n}=20$; Fig. 1B, panel d, C, panel c). Founders msd::LfngID9 (Fig. 1B, panel b, C, panel a) and ID10 (Fig. 1B, panel a) were virtually indistinguishable from msd::LfngHA3 mice (Fig. 1B, panel f, C, panel e), whereas hemizygous msd::Lfng11 mice $(\mathrm{n}=14)$ had a phenotype that was slightly less severe and more similar to homozygous msd::LfngHA2 mice (Fig. 1B, cf. panels c and e, C, cf. panels b and d). The similar external and skeletal phenotypes, as well as the irregular somites and somite patterning defects (see below) suggested that the patterning defects in msd::LfngHA embryos and mice are not caused by the HA tag and both the tagged and untagged transgene functioned equivalently.

\section{Transgene expression and phenotypic outcome}

To address whether different levels or a different timing of exogenous Lfng transcription could account for the different severity of defects in msd::LfngHA2, msd::LfngHA3, and msd::Lfng11 mice, respectively, transgene expression was assessed in day 7.5-10.5 embryos by mRNA in situ hybridization using a GFP riboprobe that detects the transgenic Lfng-GFP fusion transcripts. Fluorescence of the destabilized GFP protein was not detected. In msd::LfngHA3 embryos, transgene expression was evident already at the 1 -somite stage and 
Serth et al.

Figure 1. Structure of transgenes and external and skeletal phenotype of transgenic mice. (A) Structure of the msd::Lfng and msd::LfngHA transgenes. msd refers to the portion of the Deltal promoter directing heterologous gene expression into the paraxial mesoderm (Beckers et al. 2000). (B) Transgenic founder mice obtained with msd::Lfng (panels $a, b$ ), a hemizygous msd::Lfng11 mouse (panel $c$ ), hemizygous (panel $d$ ) and homozygous (panel e) transgenic msd::LfngHA2 mice, and a hemizygous msd::LfngHA3 mouse (panel f). (C) Skeletal preparations of a stillborn transgenic mouse obtained with female msd::Lfng founder ID9 (panel a), a hemizygous d16.5 msd::Lfng11 fetus (panel $b$ ), hemizygous (panel $c$ ) and homozygous (panel $d$ ) transgenic msd::LfngHA2, and hemizygous msd::LfngHA3 (panel e) newborn mice. Arrowheads and arrows point to fusions of neural arches and ribs, respectively.

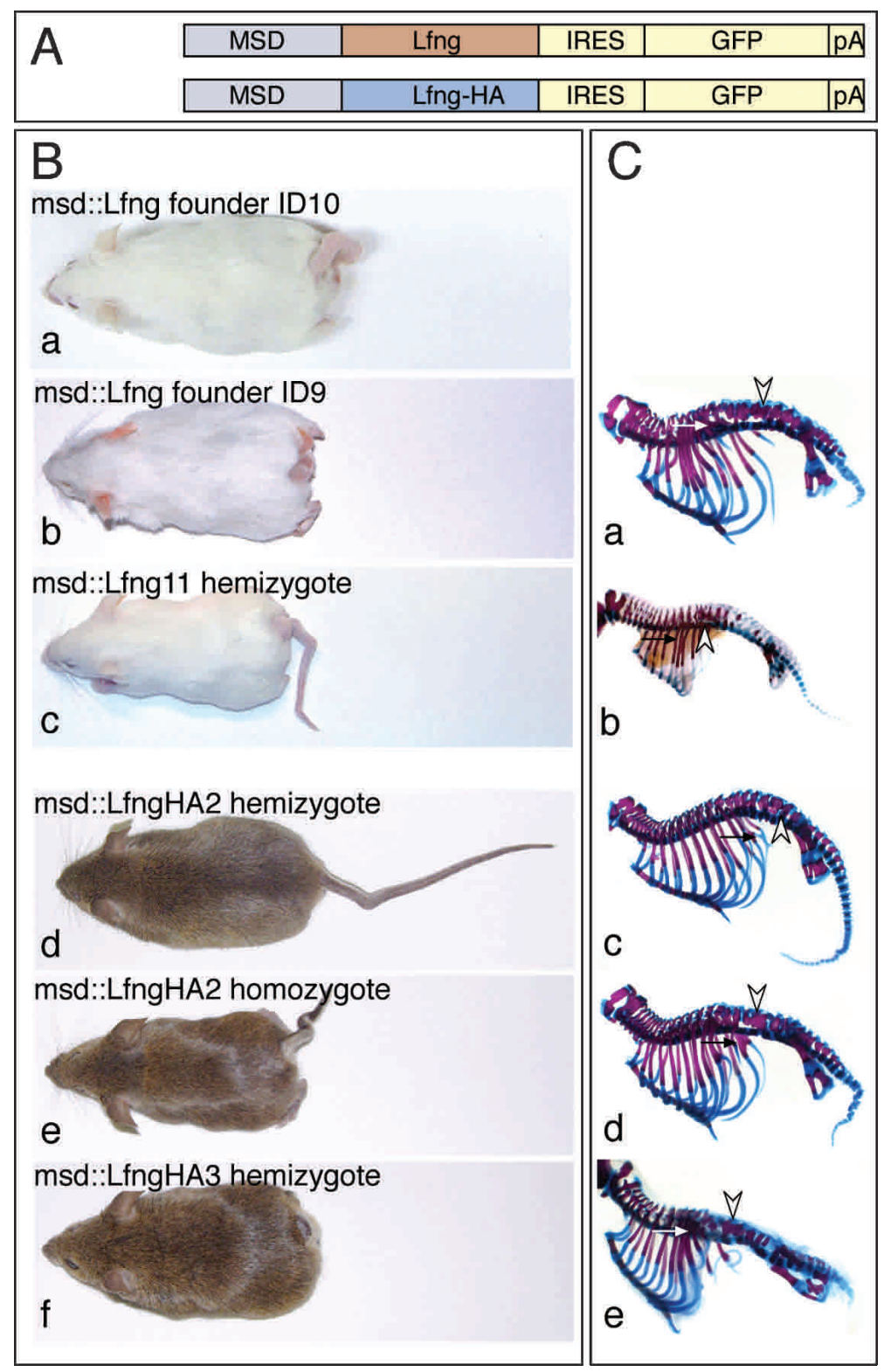

was found in the psm but not in somites of hemizygous day 8-8.5 (2-6-somite stage) embryos (Fig. 2A, panel e, B, panels a-d). Subsequently, the transgene was expressed in the psm and newly formed and differentiating somites, which were irregular in size and shape (Fig. 2A, panels $\mathrm{f}, \mathrm{g}$ ). After day 9.5, the expression domain in the psm had a sharp anterior border with lower levels of expression anteriorly (Fig. 2A, panel g, arrowheads; data not shown), which was more clearly seen in embryos after less extensive staining (Fig. 2A, panel g, inset). In contrast, hemizygous msd::LfngHA2 embryos showed only weak transgene expression in the psm but not in the somites at day 8-8.5 (Fig. 2A, panel a). Higher levels in the paraxial mesoderm were only found later in day 9.5 and older embryos (Fig. 2A, panels b,c; data not shown) in a pattern very similar to, but with levels always lower than in msd::LfngHA3 embryos. In msd::Lfng11 em- bryos, transgene expression was readily detected in the psm of day 8.5 embryos and was subsequently maintained in a pattern very similar to lines msd::LfngHA2 and msd::LfngHA3 (Fig. 2A, panels h-j). Expression levels in msd::Lfng11 embryos were between levels in msd::LfngHA2 and msd::LfngHA3 embryos. Thus, the severity of defects in the different $L f n g$ transgenic mice correlated with onset and level of transgene expression.

Transgene expression levels in the phenotypically most strongly affected line msd::LfngHA3 were examined by in situ hybridization of day 9.5 wild-type, msd::LfngHA3, and msd::LfngHA3 embryos lacking endogenous Lfng (msd::LfngHA3; Lfng ${ }^{-/}$) using a Lfng cDNA probe that detects both endogenous and exogenous mRNA. Oscillations of endogenous $L f n g$ that were evident in wild-type embryos (Fig. 2C, panels a-d) were obscured by transgenic $L f n g(\mathrm{n}=4$; Fig. 2C, panels e-h), 

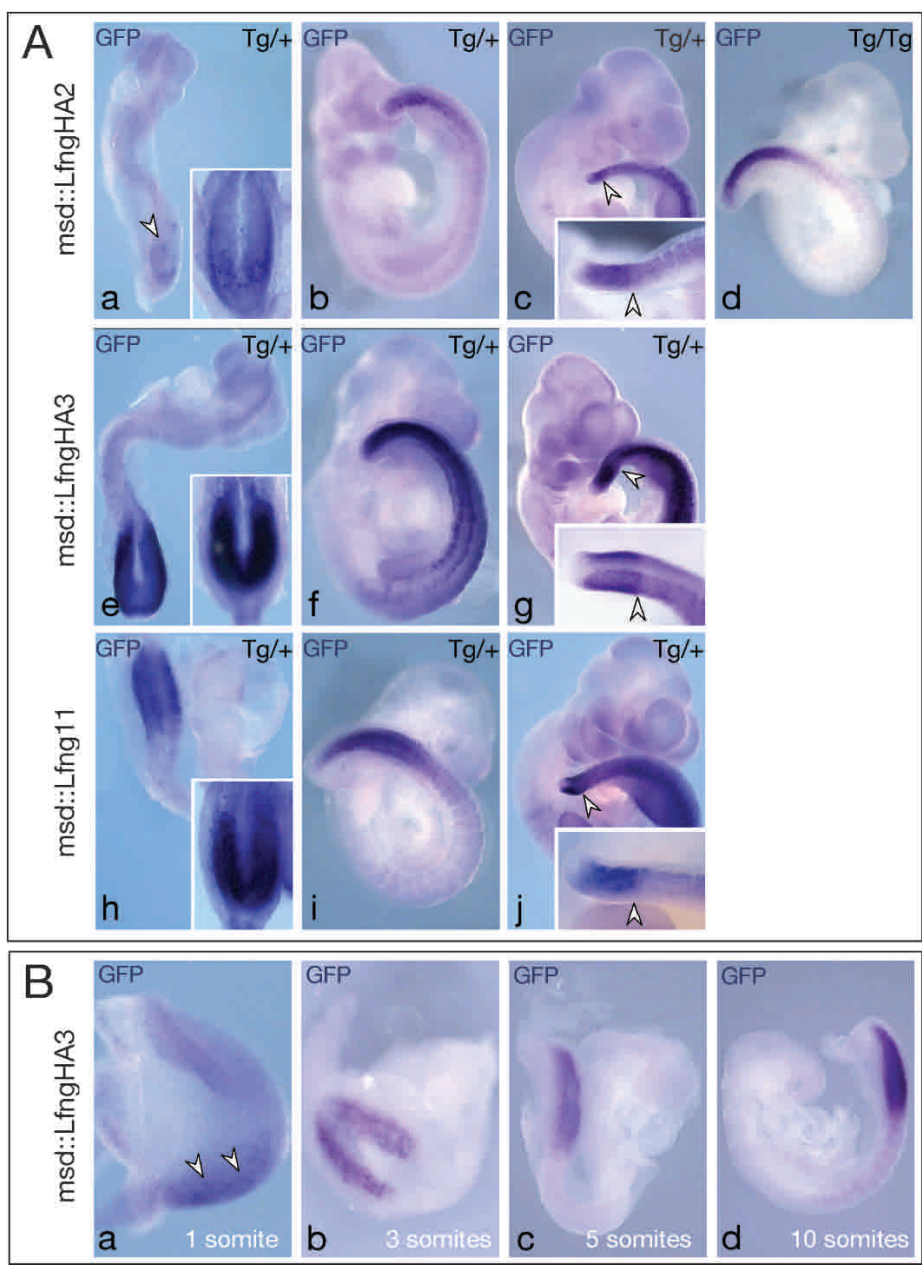

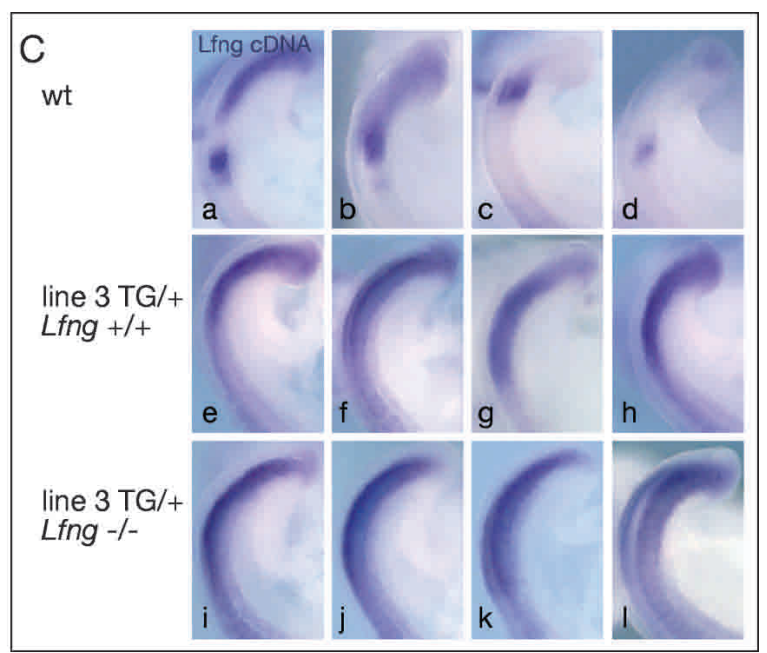

Figure 2. Transgene expression in msd::LfngHA2, msd::LfngHA3, and msd::Lfng11 embryos. (A) Wholemount in situ hybridization of day 8.5 (panels $a, e, h$ ), 9.5 (panels $b, d, f, i$ ) and 10.5 (panels $c, g, j$ ) hemizygous (panels $a-c$ ) and homozygous (panel d) msd::LfngHA2, hemizygous msd::LfngHA3 (panels $e-g$ ), and hemizygous msd::Lfng11 (panels $h-j$ ) embryos with a GFP probe detecting the exogenous $L f n g$-GFP fusion transcript. After $4 \mathrm{~h}$ color reaction, transgene expression was barely detected in the psm of day 8.5 msd::LfngHA2 (arrowhead in panel $a$ ) but strong in msd::LfngHA3 and msd::Lfng11 embryos. Insets in panels $a, e$, and $h$ show the same embryos after $21 \mathrm{~h}$ color reaction. In day 10.5 embryos, the domain of strong expression in the psm had a sharp anterior border (arrowheads in panels $c, g, i)$ and was preceded by lower levels of expression more anteriorly. Embryos shown in panels $c, g$, and $j$ were stained for $21 \mathrm{~h}$. Insets show tails after $2 \mathrm{~h}$ staining. $(B)$ Activation of the Lfng transgene in msd::LfngHA3 embryos during early somitogenesis stages. Arrowheads in panel a point to low-level expression in the psm. $(C)$ Endogenous (panels $a-d)$, endogenous and exogenous (panels $e-h$ ) and exogenous (panels $i-l)$ Lfng in day 9.5 embryos detected by a Lfng cDNA probe. Hybridizations and color reactions were done simultaneously under identical conditions.

and a similar intensity of staining was found in wildtype, msd::LfngHA3, and msd::LfngHA3; Lfng ${ }^{-/-}$( $\mathrm{n}=4$; Fig. 2C, panels i-1) embryos, suggesting that endogenous and exogenous Lfng are expressed at similar levels in this transgenic line.

To further analyze the effect of timing and level of transgene expression on phenotypic outcomes, we attempted to generate homozygous transgenic lines by interbreeding hemizygous msd::LfngHA2 and msd::LfngHA3 mice, respectively. Transgenic msd::LfngHA3 females did not reproduce, precluding the analysis of homozygous msd::LfngHA3 transgenic mice. Interbreeding of hemizygous msd::LfngHA2 transgenic mice produced some offspring with a severe phenotype similar to msd::LfngHA3 mice (Fig. 1B, cf. panels $\mathrm{d}$ and e, C, cf. panels $\mathrm{c}$ and $\mathrm{d}$ ), suggesting that they are homozygotes. Severely affected msd::LfngHA2 females, like hemizygous msd::LfngHA3 females, did not reproduce. Homozygous msd::LfngHA2 males ( $\mathrm{n}=4$, displaying a severe phenotype and homozygosity ascertained by test matings with wild-type females) mated with
msd::LfngHA2 females produced embryos half of which showed stronger transgene expression (Fig. 2A, panel d) than hemizygous msd::LfngHA2 embryos (Fig. 2A, panel b), but significantly lower Lfng expression than hemizygous msd::LfngHA3 embryos (Fig. 2A, cf. panels $\mathrm{d}$ and f). These findings suggest that constitutive expression of Lfng that moderately elevates the level of endogenous Lfng is sufficient to disrupt normal somitogenesis, and a further increase of constitutive expression has little additional effect on Notch activity.

\section{Disrupted somite patterning in msd::Lfng transgenic embryos}

Complete loss of Lfng causes irregular somite borders and disrupts somite compartmentalization as evidenced by a severely disorganized pattern of markers for anterior and posterior somite halves and affects expression of Notch pathway components (Evrard et al. 1998; Zhang and Gridley 1998). To address the effect of constitutive Lfng expression in the psm on anterior-posterior somite 
Serth et al.

patterning, we analyzed expression of Uncx4.1 and Tbx18, which mark posterior and anterior somite halves, respectively. Expression of Uncx4.1 and Tbx18 was no longer confined to anterior and posterior somite compartments, respectively, but spread throughout the somite (Fig. 3) similar to the expression in $\mathrm{Lfng}^{-/-}$mutants (Evrard et al. 1998; Zhang and Gridley 1998). In addition, the regularly spaced expression of Dll1 in posterior somite halves was disrupted, the distinct expression of D113 and Notch2 in anterior portions of newly formed somites was lost, and the anterior expression border of Notch1 was poorly defined (Fig. 3), closely resembling the somite defects found in homozygous Lfng mutant embryos (Evrard et al. 1998; Zhang and Gridley 1998). Likewise, similar to Lfng mutant embryos, Pax9 expression was diffuse, and myogenin expression domains were frequently fused in msd::LfngHA3 transgenic embryos (Fig. 3ze; data not shown). Expression of pMeso1, Mesp1, and Mesp2 were not altered in transgenic embryos (data not shown). Consistent with the significantly lower levels of transgene expression in msd::LfngHA2 embryos, disruptions of expression patterns were less severe and began at more posterior axial levels (i.e., in older em- bryos; Fig. 3j-o) than in msd::LfngHA3 or msd::Lfng11 embryos. Because transgene expression was confined to the psm of day 8.5 embryos (Fig. 2A, panel e) but somite patterning was already disrupted in msd::LfngHA3 embryos at this stage (Fig. 3p,q,r), nonoscillating Lfng expression in the psm is sufficient to disrupt anterior-posterior somite patterning, and the ectopic somitic expression at later stages is unlikely to cause these defects.

Failure of exogenous Lfng to compensate for the loss of cyclic endogenous Lfng

To test whether a reduction of cyclic endogenous Lfng modulates the phenotype caused by constitutive exogenous Lfng expression, and whether a sustained level of $L f n g$ expression can compensate for the loss of endogenous cyclic $L f n g$, msd::LfngHA3 transgenic mice also carrying the recessive $L f n g$ lac $Z$ null allele (Zhang and Gridley 1998) were generated. Mice hemizygous for msd::LfngHA3 and one copy of $L f n g^{\text {lac } Z}$ (Lfng lacZ/+/msd::Lfng) resembled hemizygous transgenic msd::LfngHA3 mice both in their external morphology and skeletal defects (26 adults and $\mathrm{n}=36$, day 15.5-16.5

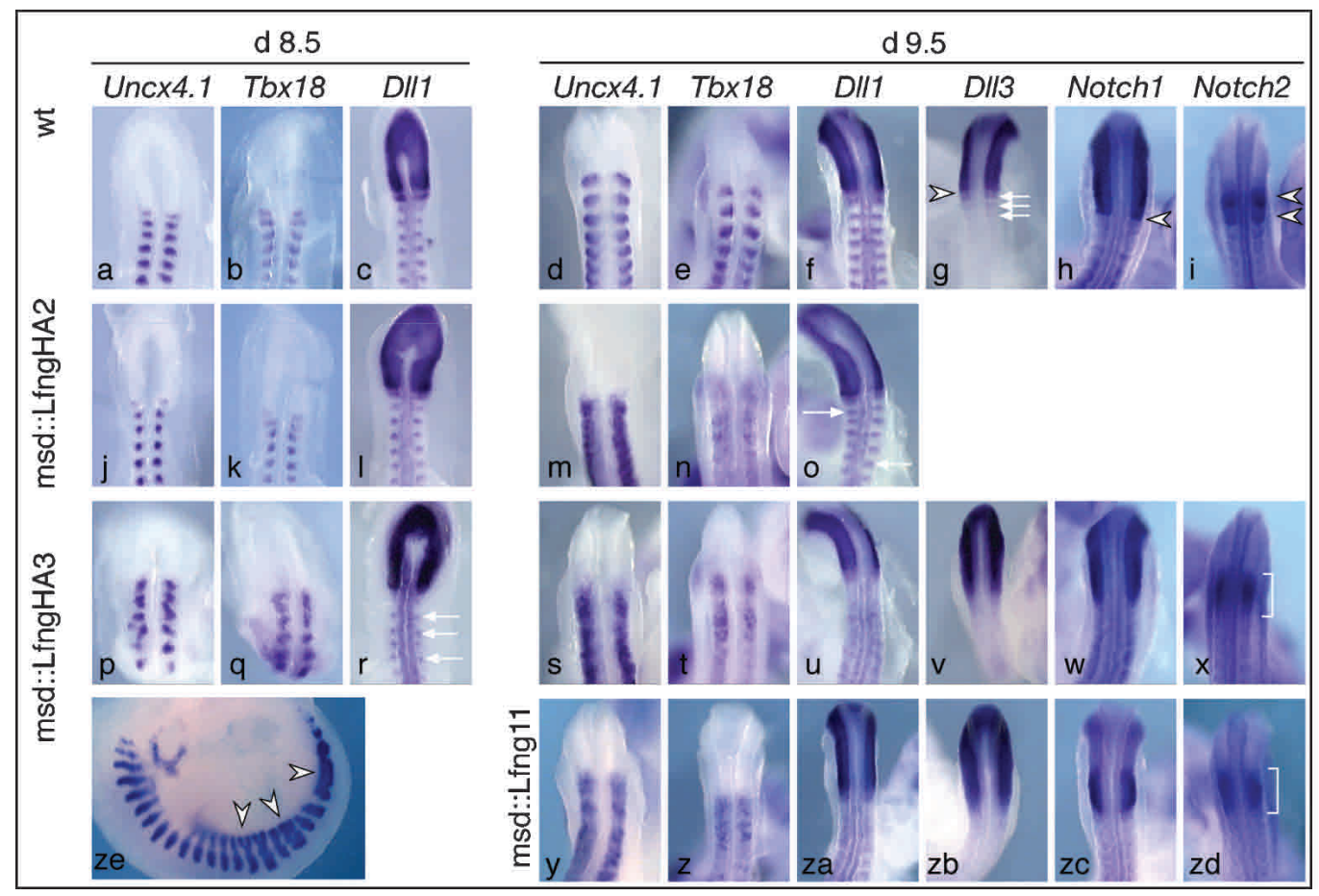

Figure 3. Somite patterning defects in msd::Lfng and msd::LfngHA transgenic mice. In situ hybridization of wild-type (a-i), msd::LfngHA2 $(j-o)$, msd::LfngHA3 $(p-x, z e)$, and msd::Lfng1 $(y-z d)$ embryos. Probes and stages are indicated above each column. In msd::LfngHA2 embryos Uncx4.1, Tbx18, and D111 expression is essentially normal in the prospective cervical somites of day 8.5 embryos but disrupted in more posterior somites of day 9.5 embryos, whereas in msd::LfngHA3 embryos expression patterns are abnormal already in day 8.5 embryos. Day 9.5 msd::Lfng11 embryos show patterning abnormalities similar to msd::LfngHA3 embryos. Arrows in $O$ and $r$ point to Dl11 expression domains out of register with the contralateral side. In msd::LfngHA3 embryos, myotome fusions (arrowheads in $z e)$ were frequently observed. $(v-X, z b-z d)$ Altered expression boundaries of Notch pathway components in day 9.5 msd::LfngHA3 and msd::Lfng11 embryos. The sharp anterior expression borders of Notch1, D113, and Notch2 in wild-type embryos (arrowheads in $g-i$ ) were indistinct and fuzzy in transgenic embryos. Expression of Dll3 in anterior somite portions of wild-type embryos (arrows in $g$ ) was not detected in transgenic embryos. The two distinct expression domains of Notch2 in wild-type embryos (arrowheads in i) were no longer discernable (brackets in $x, z d$ ). 
embryos; Fig. 4A, panels c,d,i,j). Thus, the reduction of the endogenous cyclic $L f n g$ did not significantly alter the skeletal phenotype caused by a sustained level of exogenous Lfng. Hemizygous msd::LfngHA3 mice homozygous for LfnglacZ (LfnglacZ/lacZ/msd::Lfng) displayed a phenotype virtually identical to homozygous Lfng null mutants $(\mathrm{n}=5$; Fig. 4A, panels e,f, $\mathrm{k}, \mathrm{m})$. Consistent with the comparable skeletal defects amongst msd::Lfng, msd::LfngHA3/Lfng $g^{l a c Z /+}$, and LfnglacZ/lacZ/msd::Lfng mice, expression patterns of Uncx4.1 and Dll1 were similarly altered in all these embryos (Fig. 4B; data not shown), indicating that nonoscillatory Lfng expression cannot rescue vertebral and somite malformations caused by the loss of cyclic Lfng activity. The inability of the transgene to rescue the Lfng null phenotype was not because of its lack of expression because msd::LfngHA expression in $L$ fng $^{\text {lacZ/lacZ }} / \mathrm{msd}:$ LfngHA3 mice was similar to that of msd:: LfngHA3 mice (Figs. 2C, panels i-l, 4A, panel 1). Thus, constitutive expression of $L f n g$ and absence of Lfng have a similar impact on somite formation and patterning.

\section{Effect of exogenous Lfing on cyclic gene expression in the psm}

If cyclic Lfng expression were central to the segmentation clock, noncyclic Lfng expression should disrupt oscillating gene expression. To address whether the constitutive expression of exogenous Lfng in the psm affects the periodic transcription of the endogenous Lfng gene,
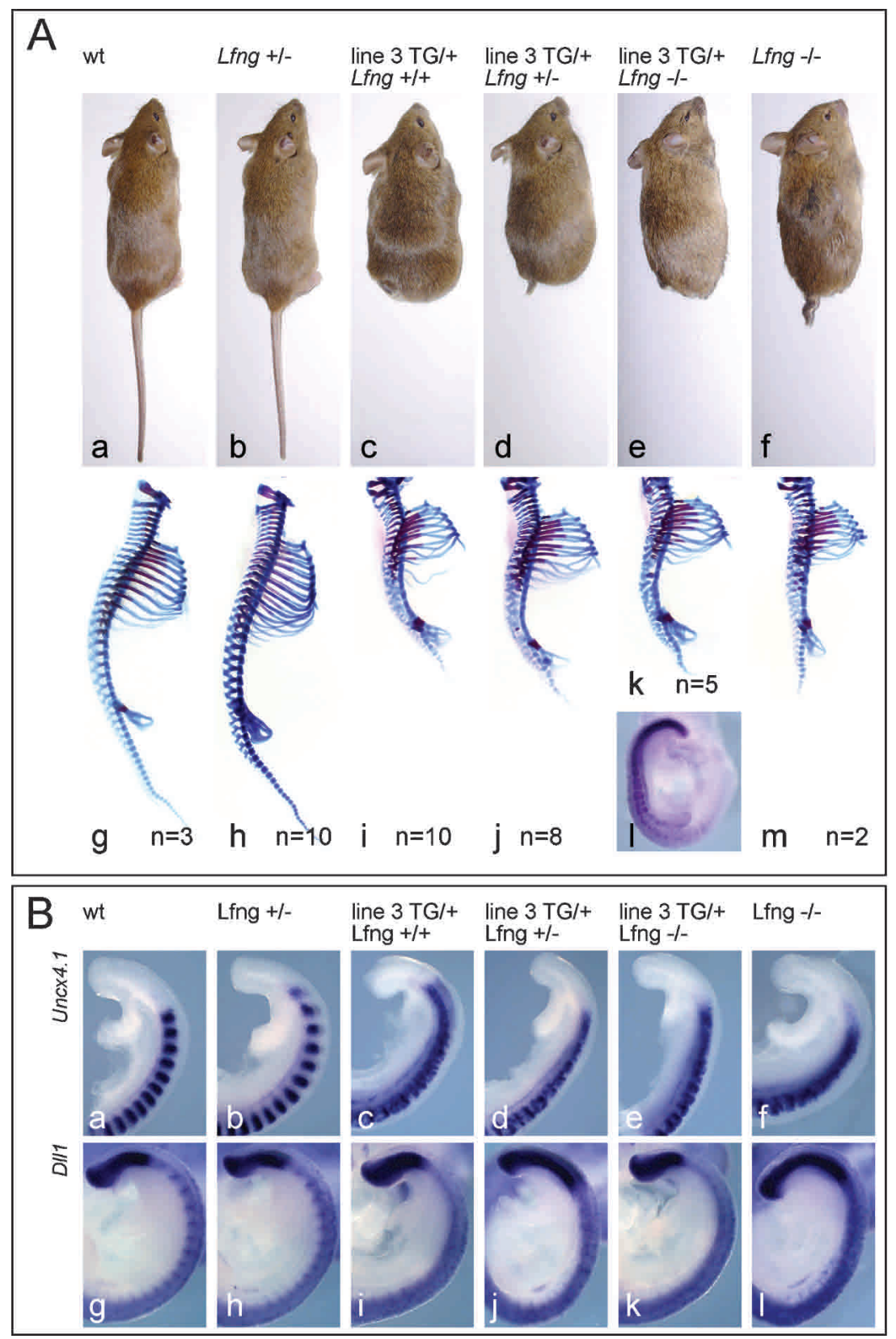

Figure 4. Vertebral column and somite patterning defects in msd::LfngHA transgenic and Lfng mutant mice. (A) External phenotypes and skeletal preparations of wild-type (panels $a, g$ ), msd::LfngHA3 (panels $c, i$ ), Lfng mutant (panels $b, f, h, m$ ), and msd::LfngHA3 transgenic mice with one (panels $d, j$ ) or both (panels $e, k$ ) copies of the endogenous Lfng gene mutated. Loss of endogenous Lfng does not alter transgene expression as detected by in situ hybridization with a GFP probe (panel 1). The number of analyzed skeletons is indicated for each genotype. $(B)$ Comparison of Uncx4.1 and Dll1 expression in wild-type (panels $a, g$ ), msd::LfngHA3 (panels $c, i$ ), Lfng mutant (panels $b, f, h, l)$, and msd::LfngHA3 transgenic mice with one (panels $d, j)$ or both (panels $e, k$ ) copies of the endogenous $L f n g$ gene mutated. Note the similarly disorganized pattern of Uncx4.1 expression (panels $c-f$ ), and the loss of Dll1 expression in posterior somite halves (panels i-1) of the different genotypes carrying the transgene. 
we used an intron probe that only detects unprocessed nuclear endogenous transcripts (Morales et al. 2002). In msd::LfngHA2 ( $\mathrm{n}=16$ ) and msd::LfngHA3 day 9.5 embryos ( $\mathrm{n}=27$ ) essentially two types of endogenous $L f n g$ expression patterns were detected in the psm. In the first type, no endogenous Lfng transcripts were detected in the posterior psm, but transcripts were present in the anterior psm in a broad band or poorly delineated stripes (msd::LfngHA2, 11/16; Fig. 5A, panels d,e; msd::LfngHA3, 20/27; Fig. 5A, panels 1,m). In the second type, endogenous $L f n g$ transcripts were present in the posterior psm, and in the anterior psm in a broad domain similar to the first group (msd::LfngHA2, 5/16; Fig. 5A, panels f,g; msd::LfngHA3, 7/27; Fig. 5A, panels n,o). Embryos with two distinct bands of expression in the anterior psm clearly resembling late phase II or phase III of the endogenous expression cycle were only observed in two embryos of msd::LfngHA2 (Fig. 5A, panel d; data not shown). This suggested that Lfng transcription is still cyclic, but the progression of Lfng oscillations and the refinement of anterior expression domains into distinct stripes are disrupted by ectopic Lfng transcripts in transgenic embryos. The broad anterior expression domain of endogenous $L f n g$ in msd::LfngHA transgenic mice was similar to the expression pattern of the lacZ mRNA in homozygous LfnglacZ mutant embryos $(\mathrm{n}=5$; Fig. 5A, panel t; Zhang and Gridley 1998). However, in contrast to transgenic embryos, lac $Z$ transcripts derived from the $L f n g^{l a c Z}$ allele, which reflect transcription of the endogenous locus, appeared down-regulated and diffuse in the posterior psm of different embryos homozygous for the Lfng ${ }^{\text {lacZ }}$ null allele (Fig. 5A, panel t; Zhang and Gridley 1998).

To directly test if endogenous transcription is dynamic in transgenic day $9.5 \mathrm{msd}:$ :Lfng embryos, posterior em- bryo portions were cut in half along the midline, one half fixed immediately, and the other half cultured for various times prior to fixation and in situ hybridization. Embryo halves from both msd::LfngHA transgenic lines showed clear differences of endogenous $L f n g$ expression in the posterior psm after $60 / \mathrm{n}=4$ and $\mathrm{n}=3$, respectively; Fig. 5A, panels i, $\mathrm{q}$ ) and $90(\mathrm{n}=3$, respectively; Fig. $5 \mathrm{~A}$, panels $\mathrm{j}, \mathrm{r}) \mathrm{min}$ of culture, and similar patterns after $\sim 2 \mathrm{~h}(\mathrm{n}=3$, respectively; Fig. 5A, panels $\mathrm{k}, \mathrm{s})$. In most cases, caudal $L f n g$ expression was down-regulated, and an anterior band of expression remained after 60 and 90 min, respectively (e.g., Fig. 5A, panel j). In three cases, expression was down-regulated in the posterior and anterior psm, and a new expression domain located caudal to the anterior stripe evident prior to culture was observed (e.g., Fig. 5A, panels i,r). In one culture, Lfng was up-regulated caudally and down-regulated in the anterior psm (Fig. 5A, panel q). These findings confirmed the results of the expression analysis of endogenous Lfng in whole embryos and indicated that in the presence of constitutive $L f n g$ activity endogenous Lfng transcription was still dynamic in the psm.

Hes 7 is essential for cyclic $L f n g$ expression in vivo, and Hes 7 expression is activated by Notch signaling (Bessho et al. 2001a,b). In Delta1 mutant embryos, Hes 7 expression was restricted to the tail bud and posterior psm, and expression patterns did not vary between embryos $(\mathrm{n}=6$; Fig. 5B, panel t), suggesting that Delta1 is required for up-regulation and dynamic expression of Hes 7 in the psm. Because Lfng potentiates Delta1-mediated Notch1 signaling in vitro (Hicks et al. 2000), we analyzed Hes 7 expression in msd::LfngHA2 and 3 embryos to address how transgene-derived constitutive $L f n g$ affects $\mathrm{Hes} 7$ expression. Hes 7 expression levels appeared not significantly altered, and variable but abnormal patterns of

\footnotetext{
Figure 5. Cyclic gene expression in msd::LfngHA2 and msd::LfngHA3 transgenic mice. (A) Endogenous Lfng expression in day 9.5 embryos detected by in situ hybridization with an intron probe. Dorsal (panels $a-g, l-O, t$ ) and lateral (panels $a^{\prime}-g^{\prime}, l^{\prime}-O^{\prime}, t^{\prime}$ ) views of the same embryos are shown. (Panels $a-c$ ) The three phases of $L$ fng expression in wild-type embryos. In transgenic embryos, essentially two types of patterns were observed. In one group of embryos (two examples are shown in panels $d, e$ and $1, m$, respectively), there was only expression in the anterior psm either in broad domains or in stripes that were in most cases poorly defined and diffuse. In the second group of embryos (two examples are shown in panels $f, g$ and $n, O$, respectively), there was a broad domain of anterior expression (white arrowheads in panels $f, g, n, O$ ), and additional expression in the posterior psm (black arrowheads in panels $f, g, n, o$ ) separated by a region of no or low expression (bars in panels $f, g, n, o)$. (Panels $h-k, p-s)$ Noncultured $\left(0^{\prime}\right)$ and cultured day 9.5 embryo tail halves (culture times indicated in the lower right corners) after in situ hybridization. Lfng expression clearly changed during 60 and 90 min of culture, and similar expression patterns were observed after $120 \mathrm{~min}$. Arrowheads in panels $i, j, q, r$ point to expression domains that changed during culture. In Lfng mutant embryos (panel $t$ ) lacZ transcripts derived from the LfnglacZ allele, which reflect transcription of the endogenous locus, were present in a broad domain in the anterior psm and appeared down-regulated and diffuse in the posterior psm of different embryos homozygous for the Lfng lacZ null allele. (B) Hes7 expression in day 9.5 wild-type, Dll1 mutant (panel $t$ ), msd::LfngHA2 (panels $d-g$ ), and msd::LfngHA3 (panels $1-O$ ) transgenic embryos. Dorsal (panels $a-g, 1-O, t)$ and lateral (panels $a^{\prime}-g^{\prime}, l^{\prime}-$ $o^{\prime}, t^{\prime}$ ) views of the same embryos are shown. (Panels $a-c$ ) The three phases of Hes7 expression in wild-type embryos. In transgenic embryos essentially two types of patterns were observed. In one group of embryos (two examples are shown in panels $d, e$ and $l, m$, respectively) there was strong expression in the posterior psm and a domain of homogenous weaker expression extending further anteriorly. In the second group of embryos (two examples are shown in panels $f, g$ and $n, o$, respectively) there was a domain of strong expression in the posterior psm (black arrowheads in panels $f, g, n, O)$ and a band of strong expression in the anterior psm (white arrowheads in panels $f, g, n, o$ ) that were separated by a variable region of weaker expression (bars in panels $f, g, n, o)$. (Panels $h-k, p-s$ ) Noncultured $\left(0^{\prime}\right)$ and cultured day 9.5 embryo tail halves (culture times indicated in the lower right corners) after in situ hybridization. Hes 7 expression changed during 60 and 90 min of culture, whereas similar expression patterns were observed after 120 min. For example, expression was up-regulated in the posterior psm (arrowheads in panels i, $q$ ), or down-regulated in the posterior psm (arrowheads in panels $j, r$ ). In Dll1 mutant embryos (panel t) Hes7 expression was confined to the posterior psm and appeared similar in all embryos. The number of embryos with each pattern or phase and the total number of analyzed embryos is indicated for each genotype.
} 


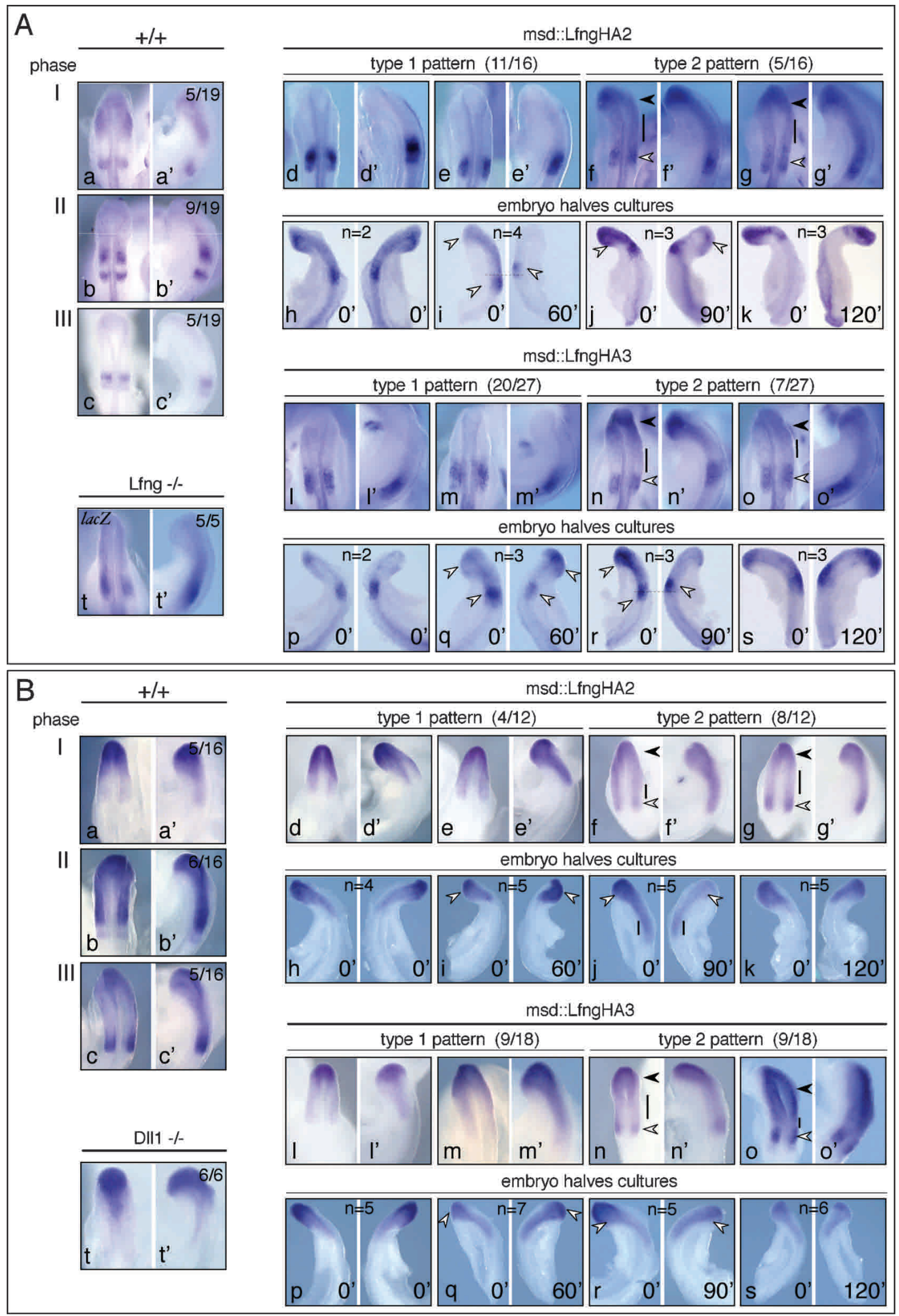

(Figure 5 legend on facing page) 
Hes 7 transcripts were found in msd::LfngHA2, and msd::LfngHA3 embryos (Fig. 5B, panels d-g, $1-0 ; n=12$ and $\mathrm{n}=18$, respectively). In one group of embryos, Hes 7 transcripts were essentially confined to the posterior half of the psm, and were most abundant in the tail bud and posteriormost psm, similar to phase I expression in wildtype embryos (msd::LfngHA2:4/12; Fig. 5B, panels d,e; msd::LfngHA3 9/18; Fig. 5B, panels $1, \mathrm{~m})$. In a second group of embryos, Hes 7 transcripts were found throughout the psm either in a fairly uniform pattern or with regions of higher expression in the anterior and posterior psm, respectively (msd::LfngHA2:8/12; Fig. 5B, panels f,g; msd::LfngHA3 9/18; Fig. 5B, panels n,o). However, no transgenic embryo showed an anterior band of Hes 7 expression that was clearly separated from the posterior expression domain by a stripe of Hes 7 nonexpressing cells and thus unambiguously resembled a wild-type phase II pattern (Fig. 5B, panel b). Thus, similar to endogenous Lfng, Hes 7 expression appeared still dynamic but was abnormal in the psm of msd::Lfng transgenic embryos. The dynamic nature of Hes 7 expression was further analyzed by culture of embryo halves. Expression patterns in noncultured and corresponding cultured embryo halves showed differences after $60(\mathrm{n}=5$ and $\mathrm{n}=7$, respectively; Fig. 5 B, panels $\mathrm{i}, \mathrm{q})$ and 90 ( $\mathrm{n}=5$, respectively; Fig. 5 B, panels $\mathrm{j}, \mathrm{r}) \mathrm{min}$ of culture, and similar expression patterns were observed after $2 \mathrm{~h}$ of culture ( $\mathrm{n}=5$ and $\mathrm{n}=6$, respectively; Fig. 5B, panels $\mathrm{k}, \mathrm{s}$ ). After 60 and $90 \mathrm{~min}$ of culture, Hes 7 expression levels were up- (Fig. 5B, panels i,q) or down-regulated (Fig. 5B, panels $\mathrm{j}, \mathrm{r})$ particularly in the posterior psm of cultured compared to noncultured embryo halves, and the extent of the expression domain in the anterior psm varied. However, the observed differences were less obvious than in the case of $L f n g$. Together, these analyses suggested that constitutive Lfng expression did not abolish dynamic Hes7 transcription but interfered with its normal pattern, implying that in wild-type embryos, cyclic Lfng is essential for the normal progression of Hes7 expression cycles.

\section{Discussion}

This study, which addresses the role of cyclic Lfng transcription in the presomitic mesoderm of mouse embryos, has shown that similar defects in somitic and vertebral patterns are found in mice that either have lost $L f n g$ function completely or have a sustained $L f n g$ activity that elevates the basal level and dampens the oscillatory activity of the endogenous $L f n g$ gene. In $L f n g$ lac Z/lac Z mice that lack endogenous $L f n g$ activity, the introduction of a noncycling transgenic $L f n g$ activity does not rescue the developmental defects, indicating that a sustained level of $L f n g$ expression cannot compensate for the loss of cyclic Lfng activity. These findings strongly suggest that oscillation of Lfng transcription in the presomitic mesoderm is essential for somite formation and patterning. Furthermore, the maintained dynamic expression of endogenous $L f n g$ transcription in the presence of constitutive exogenous $L f n g$ expression suggests that Notch signaling is not the sole determinant of cyclic transcriptional activation in the posterior presomitic mesoderm of mouse embryos.

\section{Cyclic transcriptional activation is an essential parameter of Lfng function}

Our experiments show that constitutive expression of Lfng transgenes in the psm of mice causes defects in somite border formation and anterior-posterior somite patterning and is not sufficient to compensate for the loss of cyclic endogenous Lfng. Based on the similar phenotypes in the different analyzed genotypes, we propose that transcriptional oscillation of $L f n g$ between an "ON" state and an "OFF" state is critical for Notch function in the psm (Fig. 6). In wild-type embryos, Lfng transcription cycles between its full on $\left(\mathrm{ON}_{100 \%}\right)$ and full off $(\mathrm{OFF})$ transcription (Fig. 6A, panel a) leading to transient bursts of $L f n g$ activity, which may result in waves of increased Notch sensitivity to its ligands and enhanced signaling. The loss of one copy of $L f n g$ may reduce the amplitude of the oscillations (e.g., $\mathrm{ON}_{50 \%}$ ), but expression is shut off in every cycle, which may still result in cyclic modulations of Notch activity (Fig. 6A, panel b). In msd::Lfng embryos, transgenic Lfng generates a constant level of $L f n g$ superimposed on endogenous oscillating Lfng expression (Fig. 6A, panel c). This leads to oscillations of $L f n g$ expression between two ON levels, $\mathrm{ON}_{\mathrm{HIGH}}$ and $\mathrm{ON}_{\text {LOw }}$, but prevents reaching the OFF state. Similarly, in $L f n g^{\text {lac } /+} / \mathrm{msd}:$ : Lfng embryos, endogenous Lfng oscillations with reduced amplitude are superimposed on exogenous Lfng expression (Fig. 6A, panel d). As a consequence, in transgenic embryos with one or two copies of endogenous $L f n g$, Lfng and presumably Notch activity might only alternate between two states of higher activity, $\mathrm{ON}_{\mathrm{HIGH}}$ and $\mathrm{ON}_{\mathrm{LOW}}$, and never would be able to reach below a critical threshold level or be completely OFF. In LfnglacZ/lacZ/msd::Lfng embryos, where no endogenous $L f n g$ activity is present, a constant level of exogenous $L f n g$ expression $\left(\mathrm{ON}_{\mathrm{EXO}}\right.$; Fig. 6A, panel e) might lead to permanently elevated Notch activity in the psm, whereas in homozygous null mutants there is no Lfng expression (Fig. 6A, panel f) and presumably there is no Lfng induced modulation of Notch activity. Thus, a constant low or high level of Notch activity in the psm appears not to be sufficient for somitogenesis. However, because $L f n g$ activity in the anterior psm has also been implicated in the formation of somite boundaries (Sato et al. 2002), we cannot completely rule out that disruption of normal Lfng function in the anterior psm contributes to the somite defects.

Lfng potentiates Deltal-mediated Notch signaling in vitro (Hicks et al. 2000), and Deltal signals are essential for Notch activity in the paraxial mesoderm (Hrabé de Angelis et al. 1997; Jouve et al. 2000). Thus, our findings support the idea that cyclic fluctuations of Notch signaling activity are essential for somite formation and patterning (Pourquie 1999), and Notch activity has to fall below a certain threshold during each cycle. Our findings imply that level or activity of the Lfng protein, which acts as a glycosyltransferase, oscillate in the psm. The 


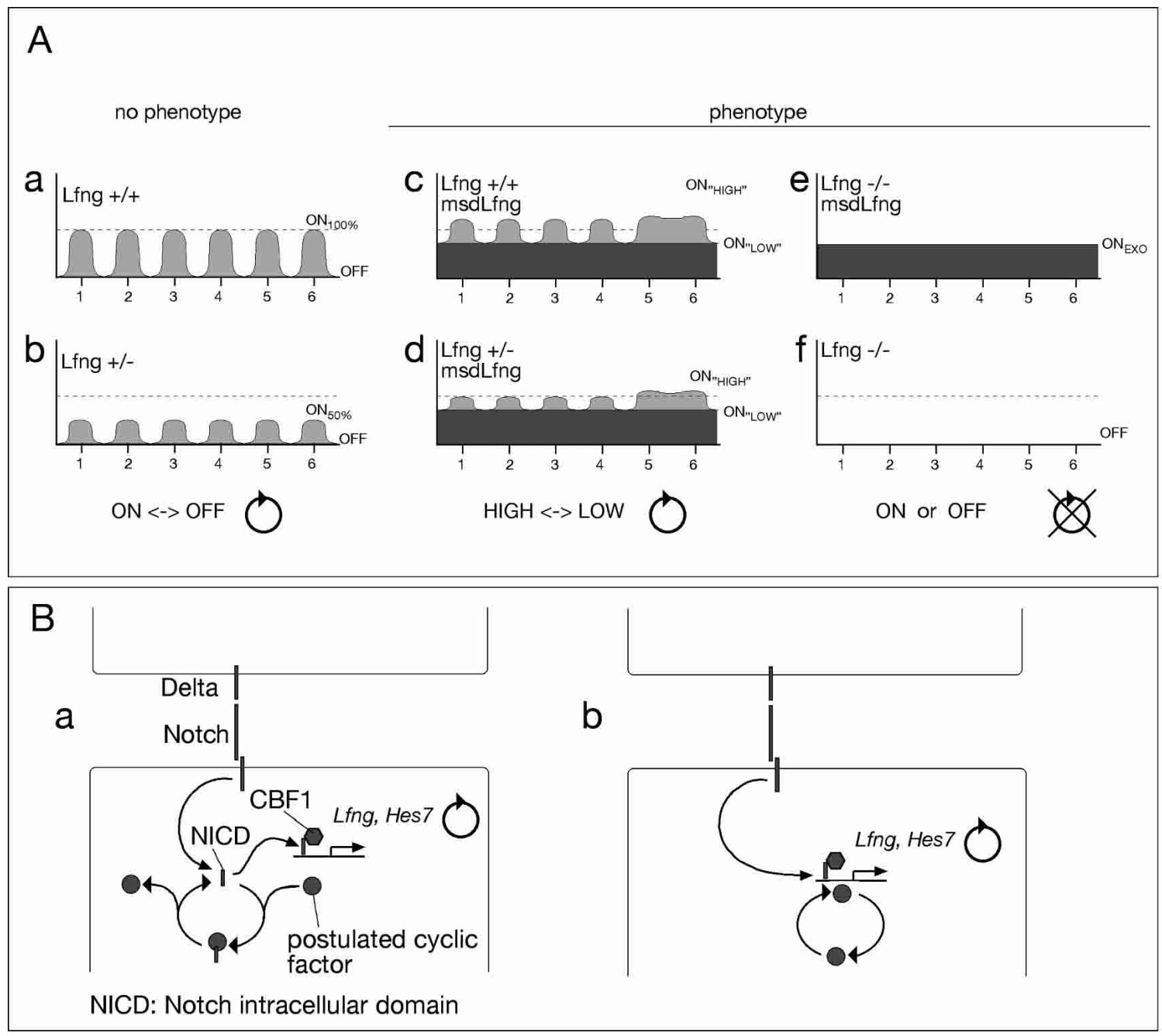

Figure 6. Scheme correlating Lfng transcription and phenotypic outcome and a model of cyclic modulation of Notch activity in the posterior psm. (A) Schematic overview of $L f n g$ transcription in different genotypes and phenotypic outcome. In wild-type (panel $a$ ) or heterozygous $L$ fng mutant (panel $b$ ) mice $L$ fng transcription cycles six times between a maximum level (wild type; ON $\mathrm{N}_{100 \%}$ ) or reduced level $\left(\mathrm{Lfng}^{+-} ; \mathrm{ON}_{50 \%}\right)$ and no expression (OFF) in groups of cells in the psm prior to these cells forming a somite. In msd::Lfng transgenic embryos (panels $c, d$ ) there is a constant level of $L f n g$ transcription superimposed on endogenous $L f n g$ that cycles in the posterior psm and is apparently noncyclic in the anterior psm, leading to alterations of $L f n g$ transcription between higher (ON $\mathrm{HIGH}_{\text {) }}$ and lower $\left(\mathrm{ON}_{\mathrm{LOW}}\right)$ levels, but $L f n g$ transcription, and presumably Lfng function, never drops below the exogenous level. In $L f n g$ null mutants with or without the transgene (panels $e, f)$ Lfng transcripts are either generated at constant levels $\left(\mathrm{ON} \mathrm{ExO}_{\mathrm{O}}\right.$ or not at all (OFF). Because the phenotypes in all embryos carrying the transgene and having no $L$ fng transcripts at all are identical, cyclic transcriptional activation of $L f n g$ appears to be essential for its function. (B) Proposed possibilities of cyclic modulation of Notch activity. (Panel $a$ ) A component that is cyclically activated binds in its active state to NICD and thus inhibits transcriptional activation of target genes. Upon inactivation of this component NICD is released and can activate target genes together with CBF1. Binding of this component could be to NICD alone or to the NICD/CBF1 complex. (Panel b) Alternatively, the component might bind in its active state to specific sequences present in the promoter of cyclic Notch target genes and acts as a coactivator.

stability and distribution of endogenous Lfng protein in the psm of mice are not known. However, Lfng protein levels fluctuate periodically in the psm of avian embryos, and overexpression of Lfng in the segmental plate of chick embryos resulted in somite defects similar to msd::Lfng transgenic mice (Dale et al. 2003), supporting our interpretation of the phenotypic consequences of constitutive Lfng expression in the psm of mouse embryos. Furthermore, our data suggest that alternating Lfng activity between moderate and high levels is not sufficient for normal somitogenesis. Lfng activity has to reach low or zero levels of activity during each cycle to enable proper somite patterning.

\section{Notch signaling and cyclic transcription in the psm}

Analyses of the Lfng promoter (Cole et al. 2002; Morales et al. 2002) and of Lfng expression in Notch pathway mutants (del Barco Barrantes et al. 1999; Dunwoodie et al. 2002; Zhang et al. 2002) have indicated that $L$ fng tran- 
scription is positively and negatively regulated by Notch signaling, Lfng activation being directly, and repression indirectly controlled by Notch (Cole et al. 2002; Morales et al. 2002). Normal transcriptional activation of $L f n g$ was disrupted in homozygous $L f$ g $^{\text {lacz/lacz }}$ embryos (Fig. 5A, panel t; Zhang and Gridley 1998) suggesting that Lfng itself is part of a feedback loop regulating its own transcription by modulating Notch sensitivity to its ligand(s). Cyclic Lfng expression was also lost in Hes $7 \mathrm{mu}-$ tant embryos (Bessho et al. 2001b) and in CBF1 (RBPjk) mutant embryos, which are likely to completely lack Notch signaling, only severely reduced noncyclic $L f n g$ expression was detected (Morales et al. 2002). Together, these results suggest that oscillating Notch signaling underlies the segmentation clock and that $L f n g$ is part of this regulatory loop. Recent experiments in chick embryos further support this model. Lfng overexpression in the segmental plate of chick embryos led to complete down-regulation of endogenous Lfng (Dale et al. 2003) similar to overexpression of a dominant-negative version of RBPjk, suggesting that in chick embryos, $L f n g$ inhibits Delta-mediated Notch activation and establishes a negative feedback loop that represents a core component of the avian segmentation clock (Dale et al. 2003).

Based on the results of this study, we propose that Notch activity is not the sole determinant of cyclic gene expression in the posterior psm of mouse embryos. Despite constitutive expression of exogenous $L f n g$ throughout the psm, we observed cyclic endogenous Lfng transcription in the posterior psm of msd::Lfng transgenic embryos. Evidence for disrupted Lfng cycling was only found in the anterior psm. The down-regulation of $L f n g$ expression in mutants with reduced or abolished Notch signaling together with the cyclic endogenous Lfng transcription in msd::Lfng transgenic embryos suggests that while Notch activity is essential for the expression of oscillating genes, cyclic Lfng and presumably cyclic Notch activity is not essential. This implies that some other as yet unidentified cyclic mechanism operates in the posterior psm and interacts with Notch signaling to initiate cyclic gene expression. This mechanism might cyclically generate or activate a component that could directly interact with activated Notch (i.e., the intracellular domain, NICD) and thereby inactivate NICD or prevent it from binding to CBF1 (Fig. 6B, panel a). An alternative possibility could be that this factor cooperates with activated Notch at the $L f n g$ promoter specifically in the posterior psm and acts as a cyclic activator or repressor (Fig. 6B, panel b). The latter possibility appears conceivable because distinct regulatory elements are required to direct cyclic $L f n g$ expression in posterior and anterior expression domains (Morales et al. 2002). Whereas our data do not allow us to distinguish between these possibilities, either mechanism would be compatible with the mutant phenotypes of Notch pathway components in mice. As long as Notch activity is present in the posterior psm, this oscillating activity could cooperate with activated Notch and induce cyclic activation of target genes. Reducing Notch signaling by removing either individual ligands or receptors would decrease tran- script levels of target genes but would not eliminate cyclic initiation of transcription on a low(er) level, as has indeed been observed (Morales et al. 2002). Total loss of Notch signaling in CBF1 mutant embryos would abolish Notch-dependent transcriptional activation altogether and consequently no cyclic activation of target genes would be possible. Also, the noncyclic expression of $L f n g$ in Hes 7 mutant embryos (Bessho et al. 2001b) is compatible with the presence of an oscillating mechanism that acts independently from, but in concert with, Notch. Hes7 is likely to repress Lfng. Thus, in the absence of $H e s 7$, cyclic repression after initiation of Lfng transcription would no longer occur, leading to the observed expression of Lfng throughout the psm in Hes7 mutant embryos. The effect of the loss of Lfng on its own expression is less clear. Loss of Lfng appears to lead to down-regulation of Lfng caudally, but it cannot be concluded with certainty that transcriptional initiation at the Lfng locus in homozygous mutants is no longer dynamic, as the lac $Z$ gene has to be used as an indicator of $L f n g$ transcription, and that transcription of the $1 a c Z$ allele with the neo gene present in the locus truly reflects the endogenous situation is not known.

Cyclic initiation of transcription of Lfng and Hes 7 in the posterior psm could trigger the establishment of a negative feedback loop that generates, reinforces, and maintains periodic Notch activity and controls the oscillating expression of cyclic genes after their initial activation. This feedback loop apparently requires both Hes 7 and Lfng because the loss of either gene disrupts oscillations of gene expression, and it can be disrupted by constitutive expression of Lfng.

Based on our results, we propose that transcriptional activation of $L f n g$ in the posterior psm of mouse embryos depends on activated Notch and an additional cyclic mechanism. This mechanism appears to be functional even with reduced Notch signaling activity, for example, in the absence of Dll1 (Morales et al. 2002), suggesting that it is independent of Notch activity and might act upstream of Notch to initiate cyclic gene expression in the posterior paraxial mesoderm. Our findings are consistent with and supported by the results of a recent study, which demonstrates that Wnt3a plays a major role in the segmentation clock, and which suggests that Wnt3a controls intracellular oscillations of Wnt/ $\beta$ catenin and Notch activity in the psm (Aulehla et al. 2003). Our data and conclusions concerning the effect of constitutive $L f n g$ expression on cyclic gene expression are at odds with the results and conclusions of Dale et al. (2003) that periodic inhibition of Notch by Lfng underlies the segmentation clock in chick embryos. Potential experimental differences that might account for the contrasting results could be different expression levels of exogenous $L f n g$ in electroporated chick and transgenic mouse embryos, or high levels of expression after electroporation already in the primitive streak or tail bud, where the msd enhancer is not or only weakly active. Future studies disrupting oscillating gene expression in defined regions of the psm and at varying levels will be required to resolve this discrepancy. 


\section{Materials and methods}

\section{Constructs and generation of transgenic mice}

The mesoderm-specific promoter msd is a 1495-bp FokI fragment fused with the minimal promoter of Dll1 containing the first exon as previously described (Beckers et al. 2000). The HAtagged Lfng cDNA was amplified with primer 1 (GGGGTACC ATGCTCCAGCGGTGCGGCCGGCGC) and primer 2 (GGG TTAACCTAGGCATAATCTGGTACATCATATGGATAGAA GATGGCGGAGCGAGGACA) to generate a KpnI site at the 5' end and an HA-tag followed by a stop codon at the $3^{\prime}$ end. The $5^{\prime} \mathrm{KpnI}$ site was used to clone $L f n g$ in frame into the first ATG codon of the msd promoter. The untagged version of the Lfng cDNA was obtained by amplifying the Lfng cDNA with the same 3' primer without the HA sequence. At the 3' end of the Lfng cDNA an internal ribosome entry site (IRES) from pIRES2EGFP (Clontech no. 6029-1) was fused in frame to a destabilized version of GFP (pd1EGFP-N1, Clontech no. 6073-1) followed by a SV40 polyadenylation signal. The whole cloning procedure of the transgene was verified by sequencing. Transgenic mice were generated by injecting the linearized construct without any vector sequences into the pronuclei of FVB fertilized eggs according to standard procedures.

\section{Genotyping of transgenic and Lfng mutant mice}

Genomic DNA was isolated either from tails of adult mice or from yolk sacs of embryos at different developmental stages. The presence of the msd::LfngHA or msd::Lfng transgene was verified by PCR using the following primers: Lfng-F7 (CCT GTCCACTTTTGGTTTGC) and Lfng-B13 (CAGAGAATGGT CCCTTGATG). Lunatic fringe mutant mice and embryos were identified with an allele-specific PCR resulting in a 500-bp PCR product for the wild-type allele with primer pair lfwF2 (CCAAGGCTAGCAGCCAATTAG) and lacZB2 (GTGCTG CAAGGCGATTAAGTT) and a 450-bp PCR product for the mutant allele with primer pair lfhs1 (GAACAAATATGGGC ATTCACTCCA) and lfgwF3 (GGTCGCTTCTCGCCAGGGC GA; Zhang and Gridley 1998).

\section{Whole-mount in situ hybridization}

Whole-mount in situ hybridizations were performed following a standard procedure with digoxygenin-labeled antisense riboprobes (Wilkinson 1992) with minor modifications using an InsituPro (Intavis AG no. 10.000) for automated in situ detection. The probes used were D111 (Bettenhausen et al. 1995), D113 (Kusumi et al. 1998), Hes7 (Bessho et al. 2001b), Hey2 (Leimeister et al. 2000), Lfng (Zhang and Gridley 1998), Lfng Intron (Morales et al. 2002), pMesogenin (Yoon and Wold 2000), Mesp 1 (Saga et al. 1996), Mesp2 (Saga et al. 1997), Myogenin (Montarras et al. 1991), Notch1 (Conlon et al. 1995), Notch2 (Weinmaster et al. 1992), paraxis (Burgess et al. 1995), Pax1 (Deutsch et al. 1988), Pax9 (Neubüser et al. 1995), Tbx18 (Kraus et al. 2001), and Uncx4.1 (Mansouri et al. 1997). A GFP-specific riboprobe was generated from a BamHI/NotI fragment from pdlEGFP-N1 subcloned into pBluescript II KS (Stratagene) using T7 RNA polymerase.

\section{Embryo culture}

Using a fine tungsten needle the caudal part of day 9.5 mouse embryos was divided into two halves along the midline in 100\% FCS as previously described (Aulehla and Johnson 1999). One half was fixed immediately, the other was cultured in a hanging drop of DMEM/F12 medium 1:1 containing 10\% fetal bovine serum at $37^{\circ} \mathrm{C}$ and $5 \% \mathrm{CO}_{2}$ for 60,90 , and $120 \mathrm{~min}$, respectively. After overnight fixation in $4 \%$ paraformaldehyde, explants were processed for whole-mount in situ hybridization as described above.

\section{Skeletal preparation of newborn and day 15.5 embryos}

Staining of the skeletons of newborn mice was performed as previously described (Zachgo et al. 1998). Day 15.5 embryos were fixed in $95 \%$ ethanol at least overnight. Then, the cartilage staining was done for $2 \mathrm{~d}$ in Alcian blue solution $(150 \mathrm{mg} / \mathrm{L}$ Alcian blue $8 \mathrm{GX}$ in $80 \%$ ethanol $/ 20 \%$ acetic acid). Embryos were rinsed and postfixed overnight again in $95 \%$ ethanol. Initial clearing was done with $2 \% \mathrm{KOH}$ for $1-2 \mathrm{~h}$ at room temperature. With Alizarin red (50 mg/L Alizarin red S in 5\% KOH) bones were stained overnight at room temperature. A second clearing was performed using $1 \% \mathrm{KOH}$ until the soft tissues became transparent. Following this incubation, the embryos were stepwise transferred to $40 \%$ glycerol.

\section{Acknowledgment}

We thank Tom Gridley for the generous gift of LfnglacZ mutants; David Ish-Horowicz and Aixa Morales for the Lfng intron probe; Olivier Pourquié for communicating results prior to publication; Andreas Kispert, Tom Gridley, Barbara Wold, Ryiochiro Kageyama, and Manfred Gessler for probes; and Johannes Beckers, Tom Gridley, Bernhard Herrmann, Andreas Kispert, and Patrick Tam for critical comments and discussion. This work was supported by the German Research Council (DFG; SFB271).

The publication costs of this article were defrayed in part by payment of page charges. This article must therefore be hereby marked "advertisement" in accordance with 18 USC section 1734 solely to indicate this fact.

\section{References}

Aulehla, A. and Johnson, R.L. 1999. Dynamic expression of lunatic fringe suggests a link between notch signaling and an autonomous cellular oscillator driving somite segmentation. Dev. Biol. 207: 49-61.

Aulehla, A., Wehrle, C., Kemler, R., Mallo, M., Gossler, A., Kanzler, B., and Herrmann, B.G. 2003. Wnt acts upstream of Notch in the segmentation clock controlling somitogenesis. Dev. Cell (In press).

Beckers, J., Caron, A., Hrabe de Angelis, M., Hans, S., CamposOrtega, J.A., and Gossler, A. 2000. Distinct regulatory elements direct Deltal expression in the nervous system and paraxial mesoderm of transgenic mice. Mech. Dev. 95: 2334.

Bessho, Y., Miyoshi, G., Sakata, R., and Kageyama, R. 2001a. Hes7: A bHLH-type repressor gene regulated by Notch and expressed in the presomitic mesoderm. Genes Cells 6: 175185.

Bessho, Y., Sakata, R., Komatsu, S., Shiota, K., Yamada, S., and Kageyama, R. 2001b. Dynamic expression and essential functions of Hes7 in somite segmentation. Genes \& Dev. 15: 2642-2647.

Bettenhausen, B., Hrabé de Angelis, M., Simon, D., Guenet, J.L., and Gossler, A. 1995. Transient and restricted expression during mouse embryogenesis of Dll1, a murine gene closely related to Drosophila delta. Development 121: 2407-2418.

Burgess, R., Cserjesi, P., Ligon, K.L., and Olson, E.N. 1995. 
Paraxis: A basic helix-loop-helix protein expressed in paraxial mesoderm and developing somites. Dev. Biol. 168: 296-306.

Cole, S.E., Levorse, J.M., Tilghman, S.M., and Vogt, T.F. 2002. Clock regulatory elements control cyclic expression of lunatic fringe during somitogenesis. Dev. Cell 3: 75-84.

Conlon, R.A., Reaume, A.G., and Rossant, J. 1995. Notch1 is required for the coordinate segmentation of somites. Development 121: 1533-1545.

Dale, J.K., Maroto, M., Dequeant, M.-L., Malapert, P., McGrew, M., and Pourquie, O. 2003. Periodic Notch inhibition by lunatic fringe underlies the chick segmentation clock. $\mathrm{Na}$ ture 421: 275-278.

del Barco Barrantes, I., Elia, A.J., Wünsch, K., Hrabe De Angelis, M., Mak, T.W., Rossant, R., Conlon, R.A., Gossler, A., and de la Pompa, J.-L. 1999. Interaction between L-fringe and Notch signalling in the regulation of boundary formation and posterior identity in the presomitic mesoderm of the mouse. Curr. Biol. 9: 470-480.

Deutsch, U., Dressler, G.R., and Gruss, P. 1988. Pax 1, a member of a paired box homologous murine gene family, is expressed in segmented structures during development. Cell 53: 617-625.

Dunwoodie, S.L., Clements, M., Sparrow, D.B., Sa, X., Conlon, R.A., and Beddington, R.S. 2002. Axial skeletal defects caused by mutation in the spondylocostal dysplasia/pudgy gene Dll3 are associated with disruption of the segmentation clock within the presomitic mesoderm. Development 129: 1795-1806.

Evrard, Y.A., Lun, Y., Aulehla, A., Gan, L., and Johnson, R.L. 1998. Lunatic fringe is an essential mediator of somite segmentation and patterning. Nature 394: 377-381.

Forsberg, H., Crozet, F., and Brown, N.A. 1998. Waves of mouse lunatic fringe expression, in four-hour cycles at two-hour intervals, precede somite boundary formation. Curr. Biol. 8: 1027-1030.

Gessler, M., Knobeloch, K.-P., Helisch, A., Amann, K., Schumacher, N., Rohde, E., Fischer, A., and Leimeister, C. 2002. Mouse gridlock: No aortic coarctation or deficiency, but fatal cardiac defects in Hey $2^{-/-}$mice. Curr. Biol. 12: 16011604.

Hicks, C., Johnston, S.H., diSibio, G., Collazo, A., Vogt, T.F., and Weinmaster, G. 2000. Fringe differentially modulates Jagged1 and Delta1 signalling through Notch1 and Notch2. Nat. Cell Biol. 2: 515-520.

Holley, S.A., Julich, D., Rauch, G.J., Geisler, R., and NussleinVolhard, C. 2002. her1 And the notch pathway function within the oscillator mechanism that regulates zebrafish somitogenesis. Development 129: 1175-1183.

Hrabé de Angelis, M., McIntyre II, J., and Gossler, A. 1997. Maintenance of somite borders in mice requires the Delta homologue D111. Nature 386: 717-721.

Ishibashi, M., Ang, S.-L., Shiota, K., Nakanishi, S., Kageyama, R., and Guillemot, F. 1995. Targeted disruption of mammalian hairy and enhancer of split homolog-1 (HES-1) leads to up-regulation of neural helix-loop-helix factors, premature neurogenesis, and severe neural tube defects. Genes \& Dev. 9: 3136-3148.

Jen, W.C., Wettstein, D., Turner, D., Chitnis, A., and Kintner, C. 1997. The Notch ligand, X- $\Delta-2$, mediates segmentation of the paraxial mesoderm in Xenopus embryos. Development 124: $1169-1178$.

Jen, W., Gawantka, V., Pollet, N., Niehrs, C., and Kintner, C. 1999. Periodic repression of notch pathway genes governs the segmentation of xenopus embryos. Genes \& Dev. 13: $1486-1499$.
Jiang, Y.J., Aerne, B.L., Smithers, L., Haddon, C., Ish-Horowicz, D., and Lewis, J. 2000. Notch signalling and the synchronization of the somite segmentation clock. Nature 408: 475479.

Jouve, C., Palmeirim, I., Henrique, D., Beckers, J., Gossler, A., IshHorowcz, D., and Pourquié, O. 2000. Notch signaling is required for cyclic expression of the hairy-like gene HES1 in the presomitic mesoderm. Development 127: 1421-1429.

Kraus, F., Haenig, B., and Kispert, A. 2001. Cloning and expression analysis of the mouse T-box gene Tbx18. Mech. Dev. 100: 83-86.

Kusumi, K., Sun, E.S., Kerrebrock, A.W., Bronson, R.T., Chi, D.-C., Bulotsky, M.S., Spencer, J.B., Birren, B.W., Frankel, W.N., and Lander, E.S. 1998. The mouse pudgy mutation disrupts $\Delta$ homologue $D 113$ and initiation of early somite boundaries. Nat. Genet. 19: 274-278.

Leimeister, C., Dale, K., Fischer, A., Klamt, B., Hrabé de Angelis, M., Radtke, F., McGrew, M.J., Pourquie, O., and Gessler, M. 2000. Oscillating expression of c-Hey2 in the presomitic mesoderm suggests that the segmentation clock may use combinatorial signaling through multiple interacting bHLH factors. Dev. Biol. 227: 91-103.

Mansouri, A., Yokota, Y., Wehr, R., Copeland, N.G., Jenkins, N.A., and Gruss, P. 1997. Paired-related murine homeobox gene expressed in the developing sclerotome, kidney, and nervous system. Dev. Dyn. 210: 53-65.

McGrew, M.J., Dale, J.K., Fraboulet, S., and Pourquie, O. 1998. The lunatic fringe gene is a target of the molecular clock linked to somite segmentation in avian embryos. Curr. Biol. 8: 979-982.

Montarras, D., Chelly, J., Bober, E., Arnold, H., Ott, M.-O., Gros, F., and Pinset, C. 1991. Developmental patterns in the expression of Myf5, MyoD, myogenin, and MRF4 during myogenesis. New Biol. 3: 592-600.

Morales, A.V., Yasuda, Y., and Ish-Horowicz, D. 2002. Periodic lunatic fringe expression during segmentation is controlled by a cyclic transcriptional enhancer responsive to Notch signalling. Dev. Cell. 3: 63-74.

Neubüser, A., Koseki, H., and Balling, R. 1995. Characterization and developmental expression of $P a x 9$, a paired-box-containing gene related to Pax1. Dev. Biol. 170: 701-716.

Ohtsuka, T., Ishibashi, M., Gradwohl, G., Nakanischi, S., Guillemot, F., and Kageyama, R. 1999. Hes1 and Hes5 as Notch effectors in mammalian neuronal differentiation. EMBO T. 18: 2196-2207.

Palmeirim, I., Henrique, D., Ish-Horowicz, D., and Pourquie, O. 1997. Avian hairy gene expression identifies a molecular clock linked to vertebrate segmentation and somitogenesis. Cell 91: 639-648.

Pourquie, O. 1999. Notch around the clock. Curr. Opin. Genet. Dev. 9: 559-565.

Saga, Y., Hata, N., Kobayashi, S., Magnuson, T., Seldin, M.F., and Taketo, M.M. 1996. MesP1: A novel basic helix-loophelix protein expressed in the nascent mesodermal cells during mouse gastrulation. Development 122: 2769-2778.

Saga, Y., Hata, N., Koseki, H., and Taketo, M.M. 1997. Mesp2: A novel mouse gene expressed in the presegmented mesoderm and essential for segmentation initiation. Genes \& Dev. 11: 1827-1839.

Sato, Y., Yasuda, K., and Takahashi, Y. 2002. Morphological boundary forms by a novel inductive event mediated by lunatic fringe and Notch during somitic segmentation. Development 129: 3633-3644.

Sawada, A., Fritz, A., Jiang, Y., Yamamoto, A., Yamasu, K., Kuroiwa, A., Saga, Y., and Takeda, H. 2000. Zebrafish Mesp family genes, mesp-a and mesp-b are segmentally expressed 
in the presomitic mesoderm, and Mesp-b confers the anterior identity to the developing somites. Development 127: 1691-1702.

Takke, C. and Campos-Ortega, J.A. 1999. her1, a zebrafish pairrule like gene, acts downstream of notch signalling to control somite development. Development 126: 3005-3014.

Weinmaster, G., Roberts, V.J., and Lemke, G. 1992. Notch2: A second mammalian Notch gene. Development 116: 931-941.

Wilkinson, D.G. 1992. Whole mount in situ hybridization of vertebrate embryos. In In situ hybridization: A practical approach (ed. D.G. Wilkinson), pp. 75-84. Oxford University Press, Oxford.

Yoon, J.K. and Wold, B. 2000. The bHLH regulator pMesogenin 1 is required for maturation and segmentation of paraxial mesoderm. Genes \& Dev. 14: 3204-3214.

Zachgo, J., Korn, R., and Gossler, A. 1998. Genetic interactions suggest that Danforth's short tail $(S d)$ is a gain-of-function mutation. Dev. Genet. 23: 86-96.

Zhang, N. and Gridley, T. 1998. Defects in somite formation in lunatic fringe-deficient mice. Nature 394: 374-377.

Zhang, N., Norton, C.R., and Gridley, T. 2002. Segmentation defects of Notch pathway mutants and absence of a synergistic phenotype in lunatic fringe/radical fringe double mutant mice. Genesis 33: 21-28. 


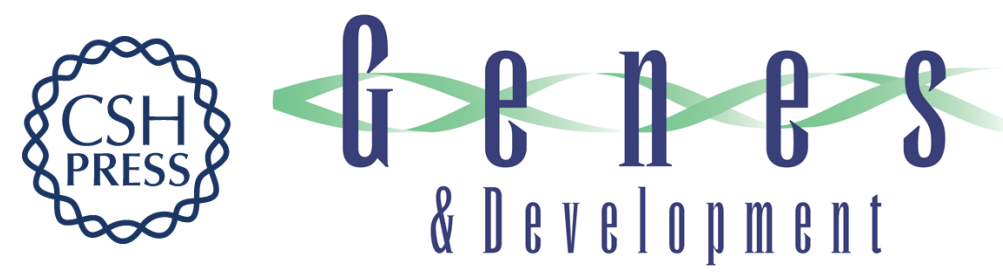

\section{Transcriptional oscillation of Lunatic fringe is essential for somitogenesis}

Katrin Serth, Karin Schuster-Gossler, Ralf Cordes, et al.

Genes Dev. 2003, 17:

Access the most recent version at doi:10.1101/gad.250603

$\begin{array}{ll}\text { References } & \text { This article cites } 44 \text { articles, } 17 \text { of which can be accessed free at: } \\ \text { http://genesdev.cshlp.org/content/17/7/912.full.html\#ref-list-1 }\end{array}$

License

Email Alerting Receive free email alerts when new articles cite this article - sign up in the box at the top Service right corner of the article or click here.

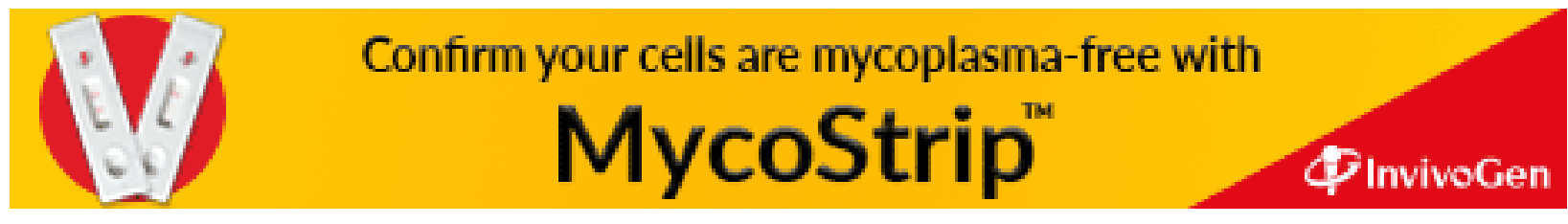

